\title{
COMPENSATING POSE UNCERTAINTIES THROUGH APPROPRIATE GRIPPER FINGER CUTOUTS
}

\author{
Adam WOLNIAKOWSKI*, Andrej GAMS**, Lilita KIFORENKO***, Aljaž KRAMBERGER**, \\ Dimitrios CHRYSOSTOMOU****, Ole MADSEN ${ }^{* * *}$, Konstantsin MIATLIUK*, Henrik Gordon PETERSEN ${ }^{* * *}$, \\ Frederik HAGELSKJ/ER ${ }^{* \star *}$, Anders Glent BUCH ${ }^{* * *}$, Aleš UDE ${ }^{* \star *}$, Norbert KRÜGER ${ }^{* \star *}$
}

\author{
*Faculty of Mechanical Engineering, Bialystok University of Technology, ul. Wiejska 45C, Białystok 15-351, Poland \\ *Department for Automation, Biocybernetics and Robotics, Jožef Stefan Institute, Jamova cesta 39, 1000 Ljubljana, Slovenia \\ *** The Maersk Mc-Kinney Moller Institute, University of Southern Denmark, Campusvej 55, DK-5230 Odense M, Denmark \\ ${ }^{* * * * *}$ Robotics and Automation Group, Aalborg University, Fibigerstraede 14, DK-9220 Aalborg East, Denmark
}

adam.wolniakowski@gmail.com, andrej.gams@ijs.si, lilita@mmmi.sdu.dk, aljaz.kramberger@ijs.si, dimi@m-tech.aau.dk, om@m-tech.aau.dk, k.miatliuk@pb.edu.pl, hgp@mmmi.sdu.dk, frederikhagel@gmail.com, anbu@mmmi.sdu.dk, ales.ude@ijs.si, norbert@mmmi.sdu.dk

received 27 October 2016, revised 19 March 2018, accepted 22 March 2018

\begin{abstract}
The gripper finger design is a recurring problem in many robotic grasping platforms used in industry. The task of switching the gripper configuration to accommodate for a new batch of objects typically requires engineering expertise, and is a lengthy and costly iterative trial-and-error process. One of the open challenges is the need for the gripper to compensate for uncertainties inherent to the workcell, e.g. due to errors in calibration, inaccurate pose estimation from the vision system, or object deformation. In this paper, we present an analysis of gripper uncertainty compensating capabilities in a sample industrial object grasping scenario for a finger that was designed using an automated simulation-based geometry optimization method (Wolniakowski et al., 2013, 2015). We test the developed gripper with a set of grasps subjected to structured perturbation in a simulation environment and in the real-world setting. We provide a comparison of the data obtained by using both of these approaches. We argue that the strong correspondence observed in results validates the use of dynamic simulation for the gripper finger design and optimization.
\end{abstract}

Key words: Grasping, Uncertainties, Gripper Design, Simulation

\section{INTRODUCTION}

The most common type of grippers utilized in automated robotic workcells (the robotic setup including the robot, the sensors (typically a vision system) and the environment) in the industry are simple parallel-finger grippers. Such grippers are relatively cheap, easy to control, and any adaptation required by changing the application at hand can often be achieved by replacing the disposable fingers. The finger design has to accommodate the shape of the object to be grasped taking into account the task constraints and the unavoidable process uncertainties, such as pose estimation errors due to shortcomings of the vision system, or variations in the objects properties.

Nowadays, this gripper design procedure usually involves empirical trials, which require substantial time, expertise, and material costs. The solution to these problems may be found in employing simulation to obtain the feedback on choices made in the design process. In our previous work, we have been interested in utilizing dynamic simulation to accelerate the design process by removing these obvious obstacles. The development of fast and accurate physics engines (a computer software that provides routines for the simulation of physical systems) over the last years, e.g. (Thulesen and Petersen, 2016), and the constant increase of the available computational power makes the use of simulation a feasible alternative to physical testing.

In this work, we first present the use case of an industrial ob- ject grasping scenario, to which we apply our simulation-based gripper optimization method introduced in (Wolniakowski et al., $2013,2015,2017)$. As compared to these works, we show in this paper the optimization process and the optimal finger geometry computed for the task context, and provide in-depth analysis of our gripper optimization method performance with regard to expected alignment of the objects. We then perform experiments to confirm our approach of using the simulation to obtain feedback on the design choices by comparing the simulated gripper performance with the real world results. Finally, we discuss the obtained results and the usage of simulation in the real-world optimization problems. We conclude with ideas on the improvement of the simulation to better reflect the real-world conditions.

\section{STATE OF THE ART}

\subsection{Gripper design in industry}

Grasping accounts for much of the workload associated with assembly, pick-and-place actions and palletizing. Still, the need of frequent setup changes associated with context switching (such as introducing a new product) provide an interesting challenge in terms of gripper design. The optimal gripper design is a persisting problem, which is currently solved by a procedure that in- 
volves a costly and time-consuming trial-and-error process. The engineering expertise necessary to produce proper finger designs is based on heuristics, collected in general guidelines, such as (Causey, 2003; Monkman et al., 2007; Wolf et al., 2005; Tarnowski, 1997; Siliano and Khatib, 2008; Carbone, 2013; Causey and Quinn, 1998). The guidelines stress the importance of minimizing gripper weight and footprint to increase the system throughput and decrease the cost, and to provide secure grasping of the handled parts.

These aims are solved by introducing gripper design features, such as cutouts (A slot in the finger surface designed to interact with the grasped objects) that embrace the grasped parts, or chamferings to reduce the gripper interference. A body of work focused on gripper design utilizing these features is present, and several overviews, such as (Boubekri and Chakraborty, 2002), have been published. Additionally, a lot of research has been devoted to the design of gripper mechanisms (Krenich, 2004; Cuadrado et al., 2004; Lanni and Ceccarelli, 2009; Ceccarelli et al., 2002). Some work has been done on the topic of gripper jaw design (Zhang et al., 2001; Zhang and Goldberg, 2006).

\subsection{Gripper learning in simulation}

The established gripper design and development process based on iterative resource and time-consuming real-world testing makes it prohibitively expensive for implementation in industrial ventures which require frequent context switching.

The usage of simulation in the design process of object aligning gripper jaws has been previously discussed in (Ellekilde and Petersen, 2006).

In (Wolniakowski et al., 2013), we have presented a general and flexible method that allows to compute an array of gripper Quality Indices based on statistical analysis of the simulated grasp outcomes. In (Wolniakowski et al., 2015) and (Wolniakowski et al., 2017), we have extended our method with additional alignment quality assessment, proposed the gripper finger parametrization, and shown an optimization procedure that yields gripper designs optimized for specific.

\section{METHODS}

\subsection{Gripper evaluation}

The gripper evaluation method based on dynamic simulation (in dynamic simulation, the object movements are calculated based on the object contacts, forces and torques, as opposed to kinematic simulation, which uses velocity and position trajectories) of grasps has been introduced and described in detail in our previous works (Wolniakowski et al., 2013, 2015, 2017). We will, however, present the key concepts here for the sake of completeness.

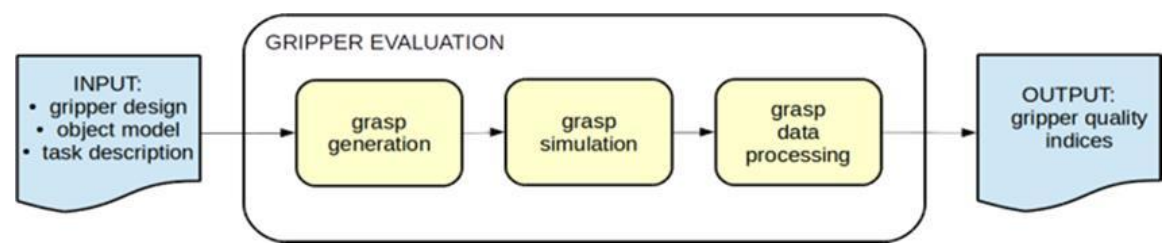

Fig. 1. Overview of the gripper evaluation procedure based on dynamic simulation of the grasping

An overview of our evaluation process is presented in Fig. 1. The input of the procedure consists of the proposed gripper design, the object model, and the task context. The context includes the description of the environment, and the grasping process to be simulated, together with the task constraints.

The evaluation process starts with the generation of a set of candidate grasps to be simulated in the surrogate scenario. The grasps are either planned, or drawn from a random distribution based on the predicted noise levels. The grasps are then executed in simulation and the statistics and the data collected. Based on these, several Quality Indices are calculated:

- Success Index (S) - quantifies the percentage of succesfully executed grasps,

- Coverage Index $(\mathrm{C})$ - describes the gripper versatility in approaching the object,

- Alignment Index (A) - describes the gripper ability to force predictable poses on the grasped object countering the pose estimation uncertainty,

- Wrench Index (W) - defines how secure the grasps executed by the gripper are,

- Robustness Index (R) - quantifies the gripper performance in the presence of process noise,

- Stress Index (T) - illustrates the structural robustness of the design,

- Volume Index $(\mathrm{V})$ - defines the cost of the gripper design (in the case of printed fingers (printed fingers are produced using additive manufacturing processes)).

\subsection{Gripper optimization}

Gripper design optimization builds on the evaluation method described above. A numerical optimization algorithm performing iterative evaluation of the gripper design paramatrized by a vector of design variables gradually approaches the best solution. In this work, we have solved the problem of optimizing the gripper cutout for the task of grasping a small metal object - the magnet. The target object and the task environment in both reality and in simulation are presented in Fig. 2.

The optimization problem is formulated as follows. The goal is to find the values of the parameters of the finger geometry, which yield the maximum value of the selected objective:

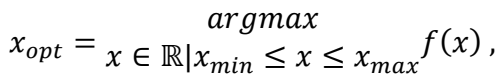

where $x$ is the vector of the design parameters, $x_{\min }$ and $x_{\max }$ are the limits on the parameter values and $f(x)$ is the objective:

$f(x)=\left(\prod_{i=1}^{n} q_{i} w_{i}\right)^{1 / \sum_{i=1}^{n} w_{i}}$,

where $q=[S, C, A, W, R, T, V]$ is the vector of Quality Indices, and $\mathbf{w}=\left[\mathrm{w}_{1}, \mathrm{w}_{2}, \ldots, \mathrm{w}_{\mathrm{n}}\right]$ is the vector of individual weights. 

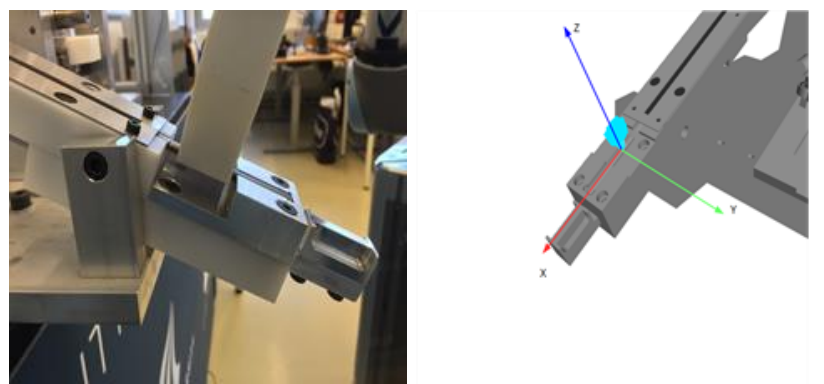

Fig. 2. The magnet grasping task: real setting (on the left) and the simulated scenario (on the right)

The Fig. 3. presents the parametrization of a simple trapezoidal cutout for the gripper finger. The vector $x$ of the design variables controls the shape of the cutout:

$x=\left[\mathrm{d}, \mathrm{w}, \mathrm{\alpha}_{1}, \alpha_{2}\right]$,

where $\mathrm{d}$ is the cutout depth, $\mathrm{w}$ is the cutout width, $\alpha_{1}$ is the cutout angle \#1, and $\alpha_{2}$ is the cutout angle \#2 (as indicated in Fig. 3). The values for the lower and upper bounds on the parameter values $x$ are presented in Tab. 1 .

Tab. 2. presents the values of the individual Quality Index weights used in this work in the computation of the objective function $f(x)$.

Tab. 1. Bounds of the design variables
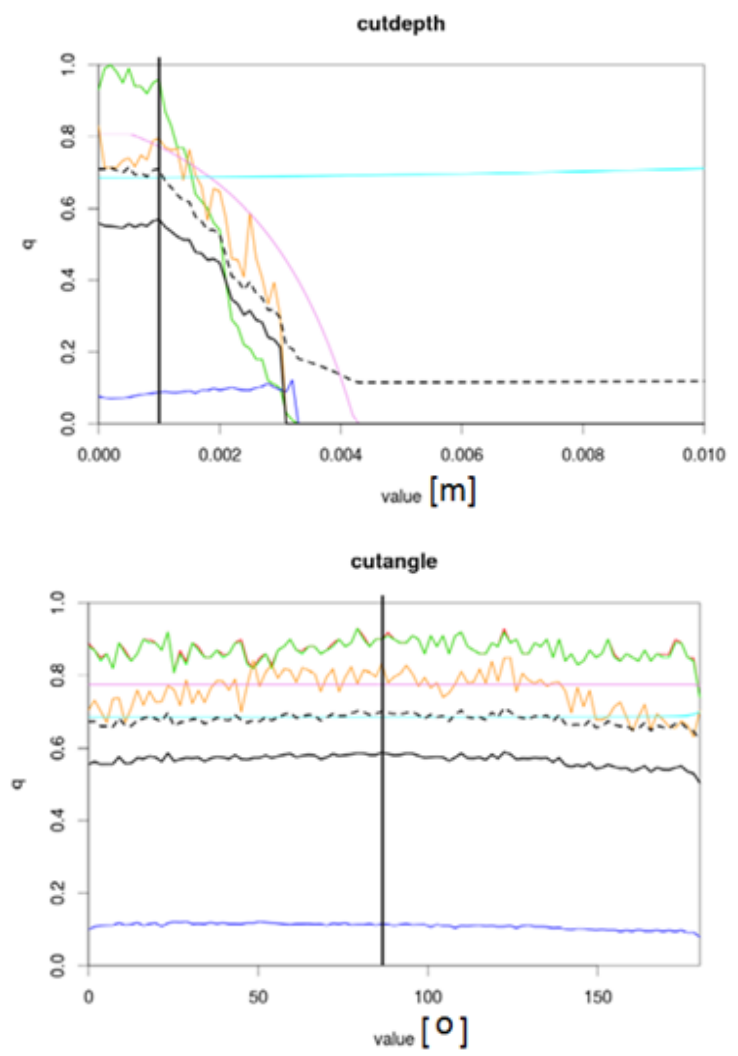

Tab. 2. Weights $w_{i}$ assigned to the individual Quality Indices in the objective function calculation

\begin{tabular}{|c|c|c|c|c|c|c|c|}
\hline QI & S & C & A & W & R & T & V \\
\hline $\mathrm{W}_{\mathrm{i}}$ & 0.1 & 0 & 1 & 0.1 & 0 & 0.01 & 0.01 \\
\hline
\end{tabular}

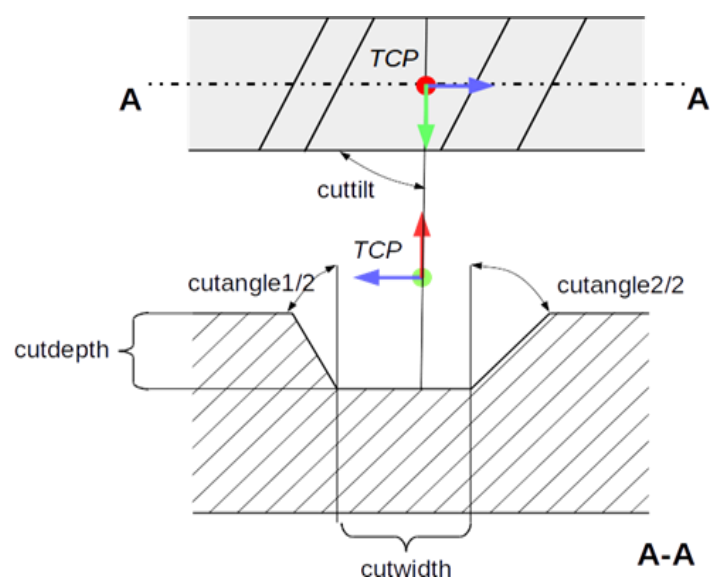

Fig. 3. The parametrization of the trapezoidal cutout. Top: the view of the inner surface of the finger. Bottom: the length-wise crossection of the finger. The coordinates are defined with respect to the TCP frame shown
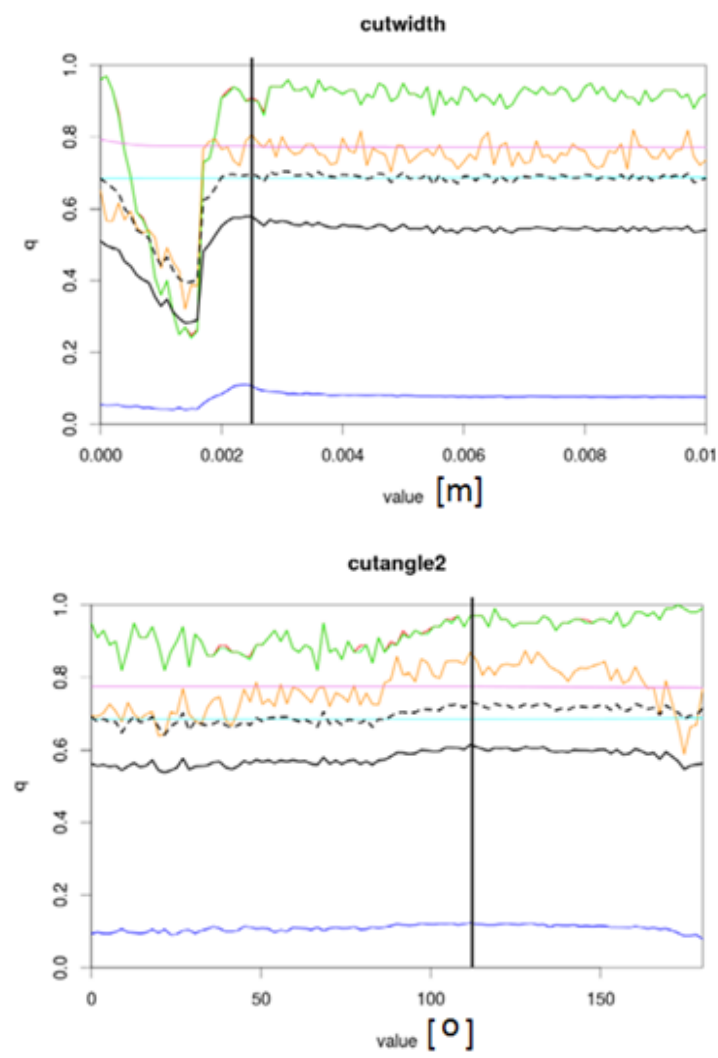

Fig. 4. The shape of the $\mathrm{Ql}$ evaluations for the trapezoidal cutout parameters during the optimization process. Top row: quality evaluation for the change in cutout depth (on the left) and cutout width (on the right). Bottom row: evaluations for cutout angle \#1 (on the left) and cutout angle \#2 (on the right). Vertical black lines indicate the optimal values chosen for the individual parameters. Green line - Success Index, orange - Alignment Index, blue - Wrench Index, solid black - $f(x)$ 


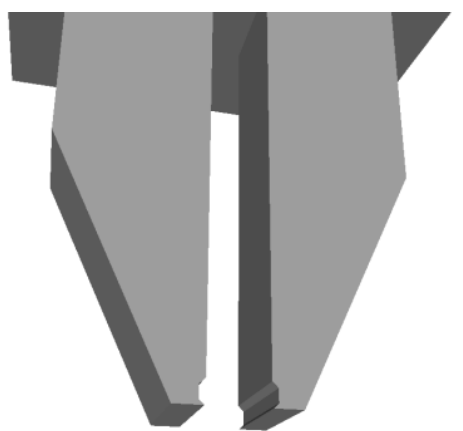

Fig. 5. The optimized trapezoidal cutout for the magnet object

In this work, we have used a simplified coordinate descent optimization method to find the optimal values for the design variables, by examining the shape of Quality Index evaluation functions across the slices of the parameter space. Fig. 4. presents the successive QI evaluations for the following order of parameters: cutout depth, cutout width, cutout angle \#1, and cutout angle \#2. The optimization criterion was to maximize in order: Alignment Index, Wrench Index, and Success Index, as reflected by the choice of weights (Tab. 2). The small weights imposed on the Stress (T) and Volume (V) objectives are in order to force the selection of a physically feasible design (in case either of these Indices equal to zero, the geometric mean would force the whole objective to 0 ).
The values chosen for the individual parameters of the design variables vector are:

$\mathrm{d}=0.001[\mathrm{~m}], \mathrm{w}=0.0024[\mathrm{~m}], \alpha_{1}=90[\mathrm{o}], \alpha_{2}=110[\underline{\mathrm{o}}]$

The optimized trapezoidal cutout shape found for the magnet is presented in Fig. 5.

\section{EXPERIMENTS}

\subsection{Setting and the protocol}

The feasibility of using simulation in order to obtain feedback used to optimize gripper designs can only be asserted if the simulation yields results comparable to those obtained from the realworld testing. After computing the optimal finger design suitable for the magnet grasping in the considered scenario, we have performed a set of experiments to confirm the validity of our approach.

We have designed a set of experiments to test whether the real and simulated grasps yield the same outcome when the reference grasp is altered with increasing offsets. Since the exhaustive exploration of the $\mathrm{SO}(3)$ pose offset space is prohibitive for such an experiment, we have decided to test and compare the offsets along the five axis of the magnet object: $y, z, R x, R y$, and $R z$, where $R$ indicates a rotation around the axis (see Fig. 2. on the right).
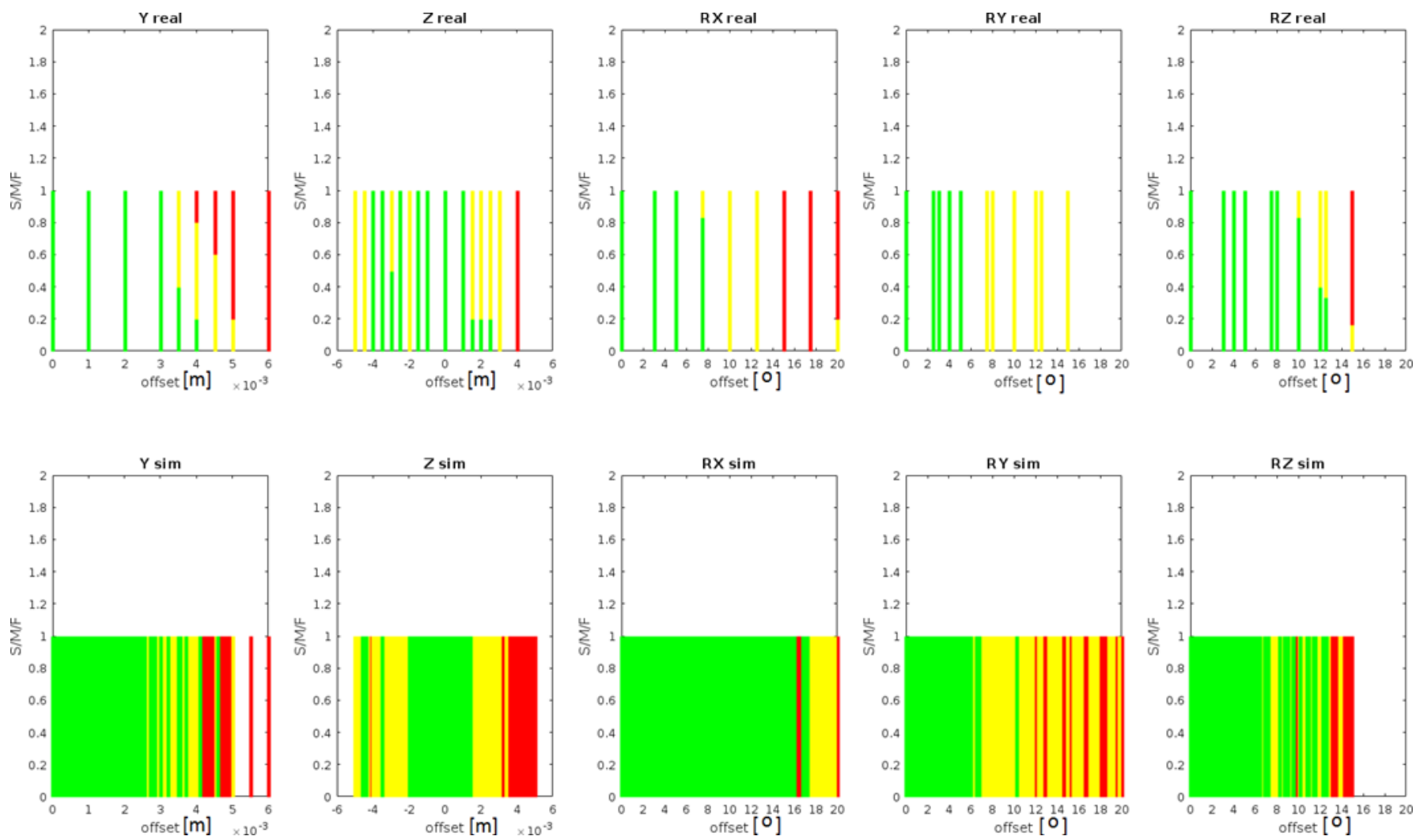

Fig. 6. The comparison of the real grasping outcomes (top) and the simulated results (bottom) - raw results. Green bars illustrate the percentage of successes, yellow - the percentage of misaligned grasps, and red - failures 

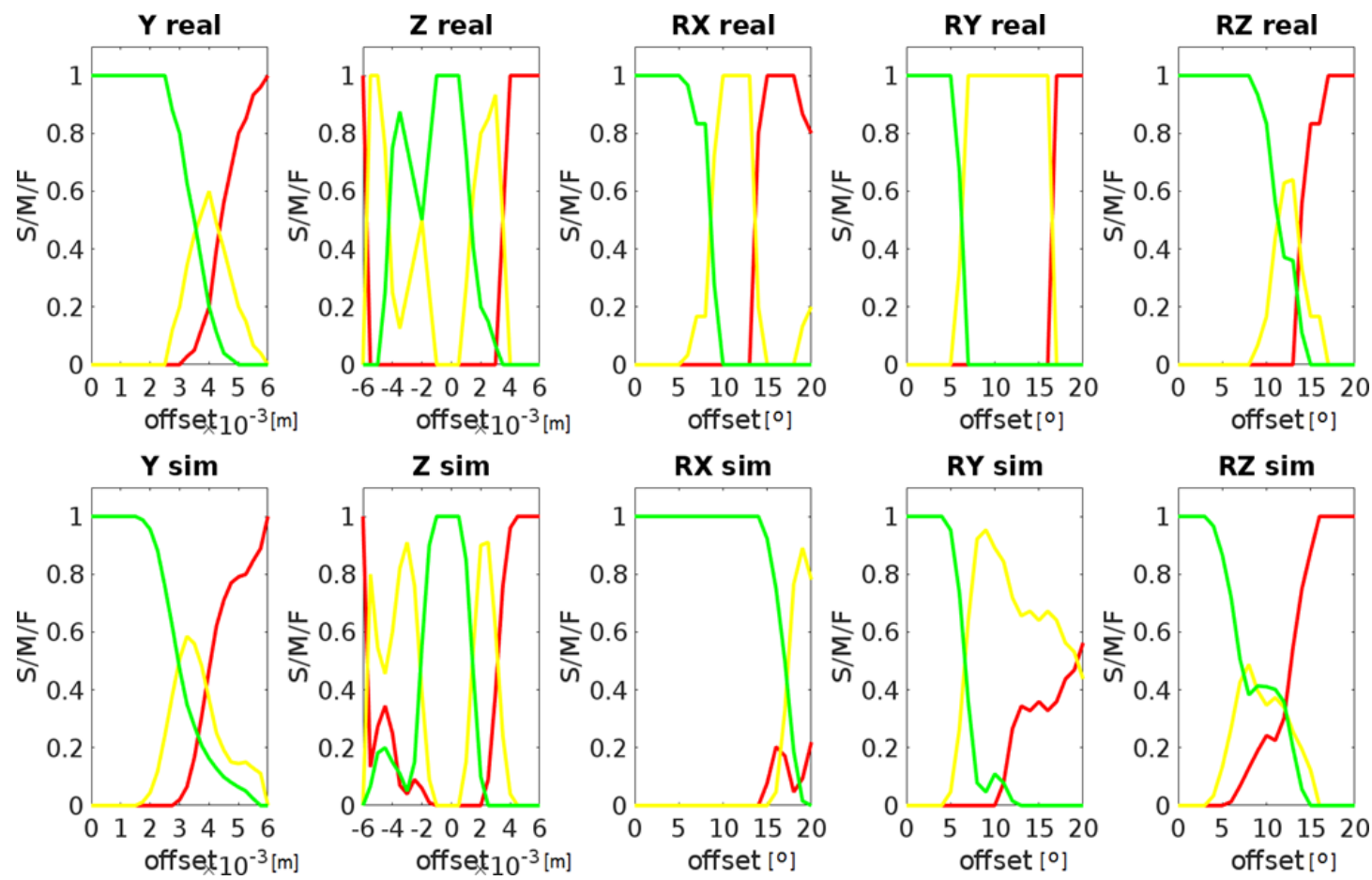

Fig. 7. The comparison of the real grasping outcomes (top) and the simulated results (bottom). Green lines illustrate the percentage of successes, yellow the percentage of misaligned grasps, and red - failures

\subsection{Results}

In the course of the performed experiments, we tested the correspondence between the results obtained from simulation and from real-world testing. The ranges of the offsets tested along the selected axes and the comparison of the outcomes is presented in Fig. 6.

Because of the fundamental difference between the stochastic nature of the real version of the experiment and the deterministic nature of the simulation, and due to the disparity in the sample coverage of the offset space, it was necessary to process the raw data obtained from the testing.

Fig. 7. presents the data processed with a median filter in order to compare the probability of grasp success in both simulation and reality. The filtering was done with a window of size $1 \mathrm{~mm}$ for the linear offsets, and 2 degrees for the angular offsets.

The results show a decent match between the simulated and the real-world outcomes. In some of the cases (Rz) the simulation yields a more conservative limit on gripper success than realworld experimentation. This can be explained by insufficient modelling of friction and compliance in our physics engine. Additionally, some artifacts have been found in the simulation results, which are due to unknown software bugs.

\section{CONCLUSION}

In this paper, we have presented the usage of a novel, simulation-based gripper optimization method in a real industrial grasping scenario. We first introduced and described our evaluation procedure, upon which the optimization method is based. We then proposed a gripper cutout parametrization, which we then optimized for a magnet grasping context. We then performed a set of experiments in both a simulated and the real-world setting, in order to confirm the validity of our approach. Finally, we discussed the obtained results.

The obtained results show a good match between the simulation and reality. The residual errors, and a number of artifacts found in simulation give us an indication of the issues and improvements that we still have to implement. Future work will involve the extension of presented methods, the fixing of aforementioned issues, and the implementation of compliance modelling.

\section{REFERENCES}

1. Boubekri N., Chakraborty P. (2002), Robotic grasping: gripper designs, control methods and grasp configurations - a review of research, Integrated Manufacturing Systems, 13, 520-531.

2. Carbone G. (2013), Grasping in robotics, Springer-Verlag London.

3. Causey G. (2003), Guidelines for the design of robotic gripping systems, Assembly Automation, 23(1), 18-28.

4. Causey G.C., Quinn R.D. (1998), Gripper design guidelines for modular manufacturing, IEEE International Conference on Robotics and Automation, 2, 1453-1458.

5. Ceccarelli M., Cuadrado J., Dopico D. (2002), An optimum synthesis for gripping mechanisms by using natural coordinates, Proceedings of the Institution of Mechanical Engineers, Part C: Journal of Mechanical Engineering Science, 2016(6), 643-653.

6. Cuadrado J., Naya M.A., Ceccarelli M., Carbone G. (2002), An optimum design procedure for two-finger grippers: a case of study, IFToMM Electronic Journal of Computational Kinematics, 15403(1).

7. Ellekilde L.-P., Petersen H.G. (2006), Design and test of object aligning grippers for industrial applications, IEEE/RSJ International Conference on Intelligent Robots and Systems, 5165-5170.

8. Krenich S. (2004), Multicriteria design optimization of robot gripper mechanisms, Solid Mechanics and lts Applications, 117, 207-218, Springer Netherlands. 
9. Lanni C., Ceccarelli M. (2009), An optimization problem algorithm for kinematic design of mechanisms for two-finger grippers, Open Mechanical Engineering Journal, 3, 49-62.

10. Monkman G., Hesse S., Steinmann R., Schunk H. (2007), Robot grippers, Wiley.

11. Siciliano B., Khatib 0. (2008), Springer handbook of robotics, Springer Verlag Berlin Heidelberg.

12. Tarnowski W. (1997), Foundations of engineering design, $C A D$, CAM, Wydawnictwa Naukowo-Techniczne, Warszawa.

13. Thulesen T.N., Petersen H.G, (2016), RobWorkPhysicsEngine: A new Dynamic Simulation Engine for Manipulation Action, IEEE International Conference on Robotics and Automation (ICRA), 20602067.

14. Wolf A., Steinmann R., Schunk H. (2005), Grippers In Motion, Springer Berlin Heidelberg.

15. Wolniakowski A., Jorgensen J.A., Miatliuk K., Petersen H.G., Krüger N. (2015), Task and Context Sensitive Optimization of Gripper Design Using Dynamic Grasp Simulation, 20th International Conference on Methods and Models in Automation and Robotics, 29-34.
16. Wolniakowski A., Miatliuk K., Gosiewski Z., Jørgensen J.A., Bodenhagen L., Petersen H.G, Krüger N. (2017), Task and Context Sensitive Gripper Design Learning Using Dynamic Grasp Simulation, Journal of Intelligent and Robotic Systems, 87(1), 15-42.

17. Wolniakowski A., Miatliuk K., Krüger N., Rytz J.A. (2013), Automatic Evaluation of Task-Focused Parallel Jaw Gripper Design, International Conference on Simulation, Modelling and Programming for Autonomous Robots, LNCS, 8810, 450-461.

18. Zhang M.T., Goldberg K. (2006), Designing robot grippers: optimal edge contacts for part alignment, Robotica, 25, 341-349.

19. Zhang T., Cheung L., Goldberg K. (2001), Shape tolerance for robot gripper jaws, IEEE/RSJ International Conference on Intelligent Robots Systems, 1782-1787.

Acknowledgement: The research leading to this publication has been funded by the EU FoF Project ReconCell (project number 680431). This research has been funded in part by the GOSTOP programme C3330-16529000, co-financed by Slovenia and EU under ERDF. 